\title{
Studying Multiscale Probabilistic and Deterministic Models for the Annotation of Cellular Networks and Tumor Metastases
}

\author{
Wilfried Allaerts \\ Biological Publishing A\&O and Immunology Department, Erasmus MC Rotterdam, Rotterdam 3000 CA, The Netherlands
}

\begin{abstract}
Comprehensive modeling of the complex behavior of cellular networks during cellular reprogramming or during tumor metastasis, has become possible by integration of different biological scales into a multiscale model. Typically, these multiscale models combine probabilistic and deterministic aspects of cellular behavior. In this paper, a review is given of modeling studies of the group of NSCLC (non-small cell lung cancers), which has a rather amalgamative appearance. Due to the heterogenous genetic background and poor predictability of divergent pathological processes, the prognosis of NSCLC to date is still very poor. The role of two important phenotypic markers, namely the TTF1 (thyroid trancription factor 1) and EGFR (epidermal growth factor receptor), are discussed with respect to disease progression, migration and metastasis potential. A strategy for introducing the cell survival perspective in tumor expansion modeling is discussed in combination with visualization techniques based on cell-state modeling, similar to cell-state modeling in reprogramming studies.
\end{abstract}

Key words: Mixed multiscale models, cell reprogramming, cell migration phenotype, non-small cell lung cancer.

\section{Introduction}

During the first decade of this millennium, a few distinguishing discoveries have changed our understanding of cellular networks and of the behaviour of tumor cell populations. First, the Nobel prize winning discoveries of John Gurdon and Shinya Yamanaka demonstrated that mature cells can be reprogrammed to become pluripotent stem cells. Yamanaka showed that only four transcription factors were needed to generate all three germ layers of embryonal development $[1,2]$. Then, new experimental evidence has been found establishing the role of stem cells in tumor development. Classically, chemotherapy has been founded on the paradigm of killing fast-growing tumor cells. In vitro studies now have ascertained that tumor cells have only limited proliferative potential, whereas another fraction of the

Corresponding author: Wilfried Allaerts, Ph.D., research field: theoretical immunology and mathematical biology. E-mail: w.allaerts@planet.nl. tumor cell population has stem-cell-like properties and gives rise to the majority of the tumor cell progeny [3]. Third, the finding that neoplastic development into metastasis may be explained in terms of an escape from homeostatic control following a cellular adaptive response to a stressful environment [4]. And finally, the microarray technology has profoundly changed the way of looking at biological data altogether. Therefore, not only NGS (Next-Generation Sequencing) techniques but a complete area of new analyis and interpretative tools became available and refurbished the research practice in this era.

Comprehensive modeling of the growth of tumor systems has become possible following the understanding that different biological scales have to be integrated into a multiscale model. These biological scales, from initial molecular mechanisms at the transcription level until the functioning of vital organs during terminal disease phases, so far are only distantly related in varying disciplines. Most promising are mixed models integrating both probabilistic and 
deterministic relations, between spatial (random) search mechanisms, dissemination routes (drainage systems), molecular dynamics and biochemical reaction pathways.

Recent studies on reprogramming of sommatic cells into iPS (induced pluripotent stem) cells have addressed the dynamics of the process of cell reprogramming. Polo et al. [5] described a pattern of bivalent domains after a first transcriptional wave and identified genes that acted as "roadblocks" in reprogramming. Shu et al. [6] presented a complex "seesaw" model of lineage specification in reprogramming. The abstract notion of a cell-state landscape directing cell lineage development is discussed in a recent paper [7].

As Edelman [8] already pointed out, incorporating cellular differentiation mechanisms, as well as activation of apoptosis and random cell migration processes into one comprehensive model for cell (lineage) development is a tedious task. Cell lineage development is well documented in the myeloid and lymphoid pathways of normal and malignant hematopoiesis [9, 10]. Recent modeling is based on pathological examples which better help to understand the complex behavior of cellular networks. In this paper, we will focus on the behavior of NSCLC, which has still a very poor prognosis for long term survival.

\section{The NSCLC (non-small cell lung cancer) Case}

\subsection{TTF1 Is Called a Proto-Oncogene in Lung Cancer}

To date, not a single marker is available to characterize all different phenotypes of NSCLC. A slight majority (approximately $55 \%$ ) of pulmonary adenocarcinomas expresses the TTF1 (thyroid transcription factor 1) [11]. Amplification of the TTF1 gene is found in $13 \%$ of ACs (adenocarcinomas) and $\sim 9 \%$ of SCCs (squamous cell carcinomas) [12].

On the other hand, TTF1 is expressed at early stages of thyroid, lung and brain development, and well before the expression of its target genes. This suggests a key role in the development of these organs. TTF1 is known as a member of the homeodomain-containing transcription factor family (reviewed by Boggaram [13]). In the thyroid, TTF1 controls the expression of the thyroglobulin [14], the thyrotropin receptor [15] and several other genes $[13,16]$. In the brain, the important growth factor nestin appears to be regulated by TTF1, via the T1 $\alpha$ promoter [17]. In the lung, however, TTF1 is essential for the expression of several members of the SP (surfactant protein) family (SP-A, SP-B, SP-C) [18-20], and regulates the expression of CCSP (Clara cell secretory protein) [21] and of the ABCA3 (ATP-binding cassette transporter A3) genes [22]. In the adult lung, TTF1 is expressed in the Type II epithelial (alveolar) cells and Clara bronchiolar epithelial cells (in bronchioles) [23] (Fig. 1).

The finding that $2 \%-13 \%$ of lung adenocarcinomas show amplification of the TTF1 gene, suggests that over-expression of TTF1 target genes in one way or another stimulates malignant cell proliferation, although the relationship of these genes with cell cycle propagation is still elusive. TTF1 is called a proto-oncogene in lung cancer [13]. According to the escape-from-homeostatic-control-hypothesis of Nguyen [4], over-expression of the TTF1 gene and its target genes may also improve the conditions for metastasing cells to survive in a stressful environment.

On the other hand, both ceramide, a product of sphingolipid breakdown, and RNAi (RNA-interference) molecules to TTF1 have blocking effects on cell cycle progression and increase apoptosis [13, 24, 25]. Ceramide is an important signaling molecule in the inflammatory response, for instance as a response to the release of mediators of acute lung injury (TNF- $\alpha$, platelet-activating factor or Fas/Apo ligand) [24]. Ceramide decreases the release of SP-B, a hydrophobic protein which is essential for surfactant function and lung stability. The inhibitory effects of ceramide and RNAi appear to be exerted at the transcriptional level, via downregulation of TTF1 DNA binding activity [13, 24]. 

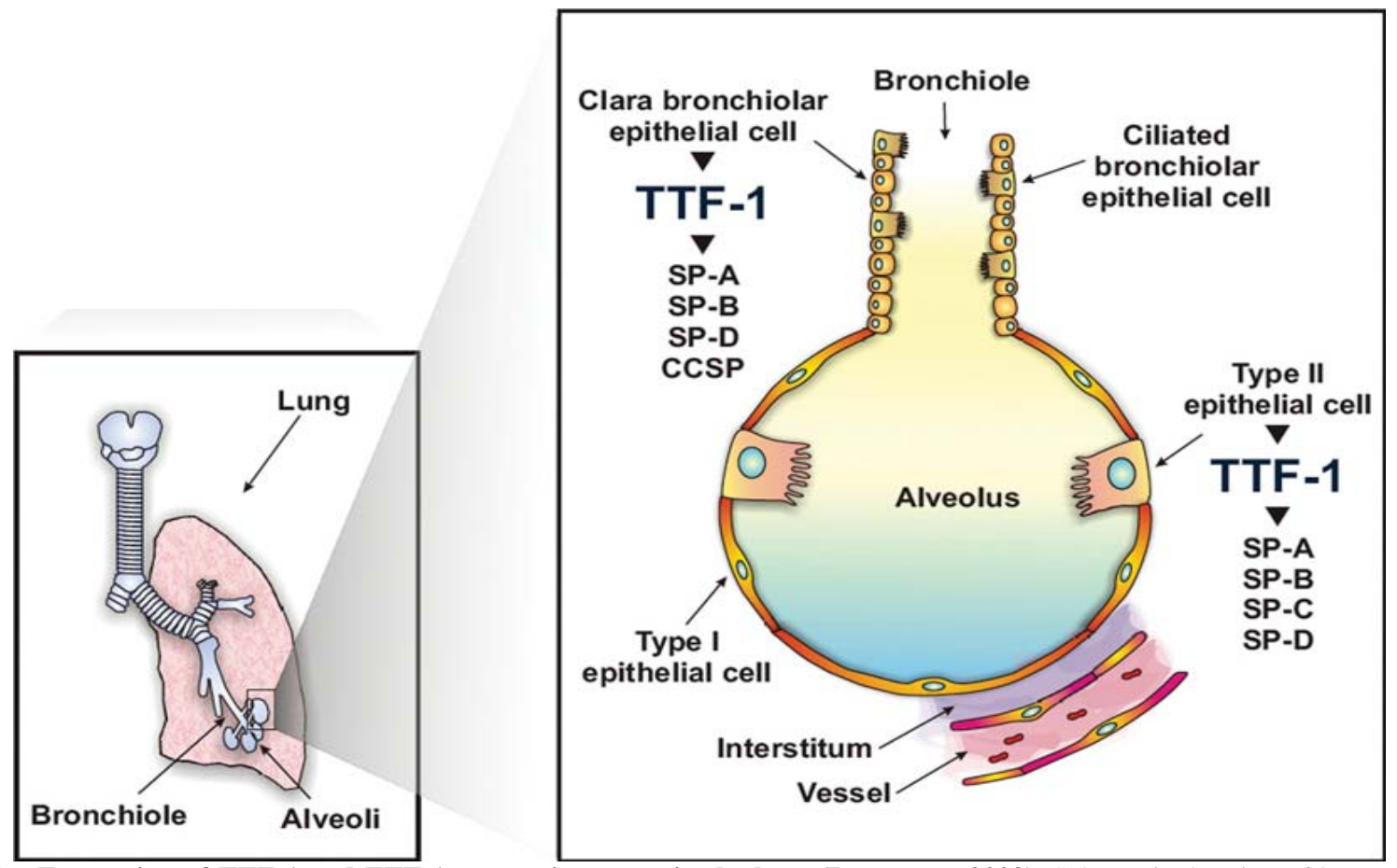

Fig. 1A Expression of TTF-1 and TTF-1-responsive genes in the lung (Boggaram 2009). Schematic drawing of lung and lung alveolus showing the locations of type I and type II cells and Clara epithelial cells. TTF-1 is expressed by alveolar type II and Clara epithelial cells. ( 2009, Biochemical Society, Clinical Science, Vol. 116, p. 28).

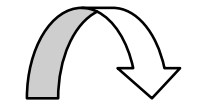

? Gene amplification in 2-12\% of lung adenocarcinoma

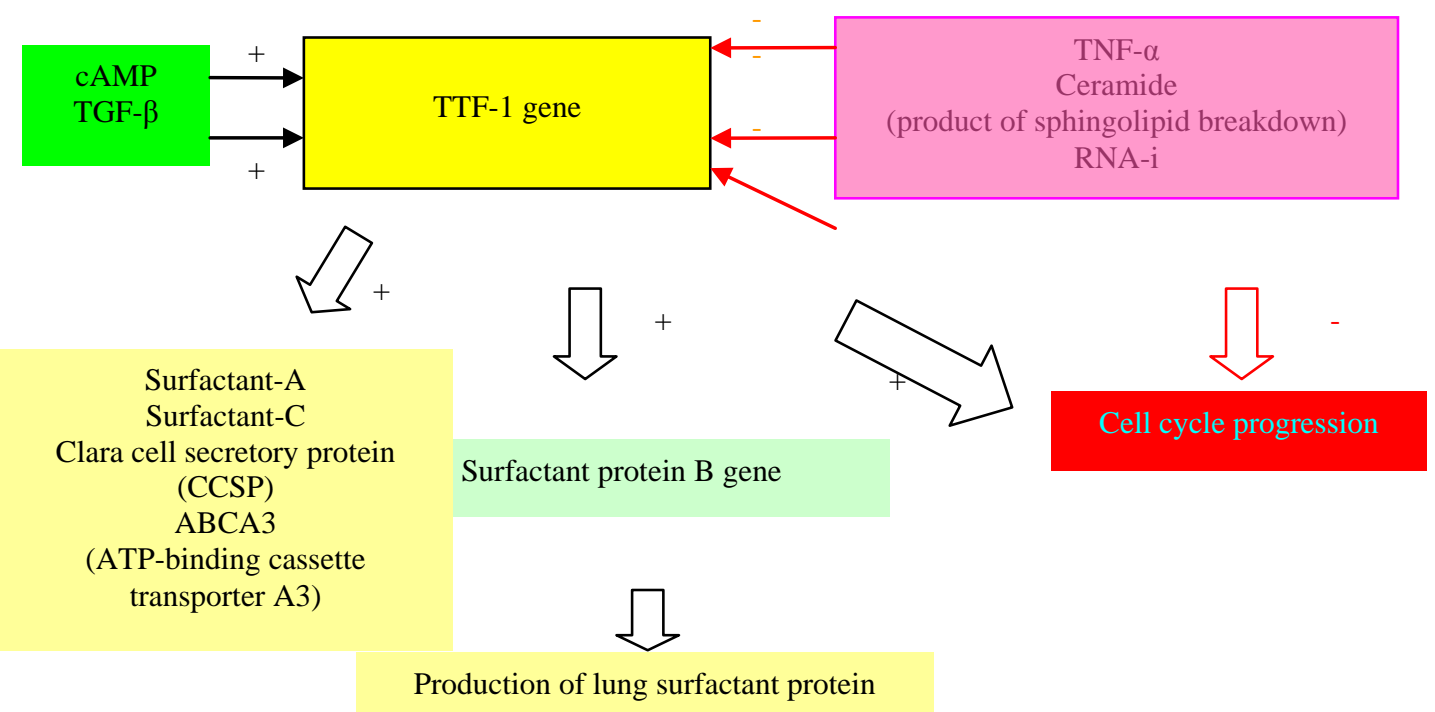

Fig. 1B Regulation of TTF-1 gene expression and of TTF-1-responsive genes (after Boggaram [13]).

Finally, recent studies have shown that alveolar Type II cells have the capability of initiating lung tumor development [26] (see section 2.2 below). Moreover, AEC2s (alveolar Type II epithelial cells) were discovered to be stem cells of the adult lung [27].
2.2 Stem Cells of the Lung and Lung Tumor Development

The role of slow-growing cancer stem cells in tumor development has been firstly described in acute 
myeloid leukaemia by John E. Dick et al. [28]. According to this "cancer stem-cell hypothesis", not all cancer cells are alike, and there exists a "pecking order" according to which an abnormal stem cell is both the key to forming and feeding a cancer. Nowadays, organ-typic stem cells have been discovered in many solid tissues, and the cancer stem cell hypothesis has been confirmed in vitro [3].

In the adult lung, these stem cell properties may be exerted by the SPC (surfactant associated protein C)-expressing alveolar Type II cells [26, 27]. In SPC ${ }^{+}$ alveolar cells, but not in $\mathrm{SPC}^{+}$BASCs (bronchioalveolar stem cells) of the bronchioalveolar duct junction, activation of the KRAS-oncogene, alone or in combination with the removal of the tumor suppressor $p 53$, resulted in development of alveolar tumors [26]. Also, using individual lineage-labeling, AEC2s were shown to self renew in vitro and differentiate over about a year in both AEC2s and cells expressing Type I markers, AEC1s (alveolar Type I epithelial cells) [27]. Moreover, after specific ablation of AEC2s, individual survivors undergo rapid clonal expansion and cell dispersal [27], proving the stem cell hypothesis for the lung alveoli. This at least corroborates the theory that NSCLC may be initiated in alveolar Type II cells [26].

\subsection{EGFR Is an Intrinsic Mitogen, Causing a Migration Dominant Phenotype}

Another factor that is frequently overexpressed in NSCLC is the EGFR (epidermal growth factor receptor)[29, 30]. Binding to the extracellular domain of EGFR, for instance by EGF (epidermal growth factor) or TGF $\alpha$ (transforming growth factor $\alpha$ ), produces several downstream effects that affect phenotypic cell behavior including proliferation, invasion, metastasis and inhibition of apoptosis [31]. A very prominent effect of these growth factors is that they lead to EGFR hyperactivity [32, 33], and thus increase tumor cell motility, invasiveness and finally enhance lung metastasis [34] (Fig. 2). Wang et al. [35] developed a multiscale model for investigating the tumor expansion dynamics of NSCLC within a two-dimensional microenvironment (in silico) (Fig. 3). They used the concentrations of EGF, glucose and the oxygen tension as external chemical cues. They found that a phase-transition from the proliferative to the migrative phenotype might occur, depending on the availability of the growth factor (EGF) and the distance to nutrient abundance. As a consequence, downstream EGFR-ERK (epidermal growth factor receptor-extracellular signal-regulated kinase) signaling (Fig. 2) may be processed more efficiently, resulting in an accelerated spatio-temporal expansion rate and increased metastasis [35].

\subsection{Routes for Lung Cancer Metastasis}

In contrast to the enormous impact on cancer-related deaths, relatively few studies have addressed the possible routes for lung cancer metastasis [36].

In current pathology textbooks, the routes for cancer metastasis are classified into basically four main types, namely (1) contiguous invasion of adjacent tissues, (2) (retrograde) lymphatic dissemination, (3) vascular invasion and (4) metastasis via coelomic cavities (e.g. pleural dissemination). In the case of lung cancer, also retrograde venous seeding is possible, most notably via the paravertebral plexus. Most routes of cancer dissemination are characterized by some fortuitous, maximum likelihood principle. For instance, lymphatic vessels lack a basement mebrane, and therefore form an easy target for dissemination. The (para) vertebral venous plexuses, because of their valveless nature may allow unmitigated tumor cell dissemination [37]. This may explain how pelvic neoplasms, e.g., carcinoma of the prostate, may metastize in vertebral bodies: the tumor cells spread into the internal vertebral plexuses via their connections with the pelvic veins. This especially occurs when the blood flow is temporarily reversed by raised intra-abdominal pressure or postural alterations [38]. A similar route would be involved in metastases from breast, renal and lung cancers. 


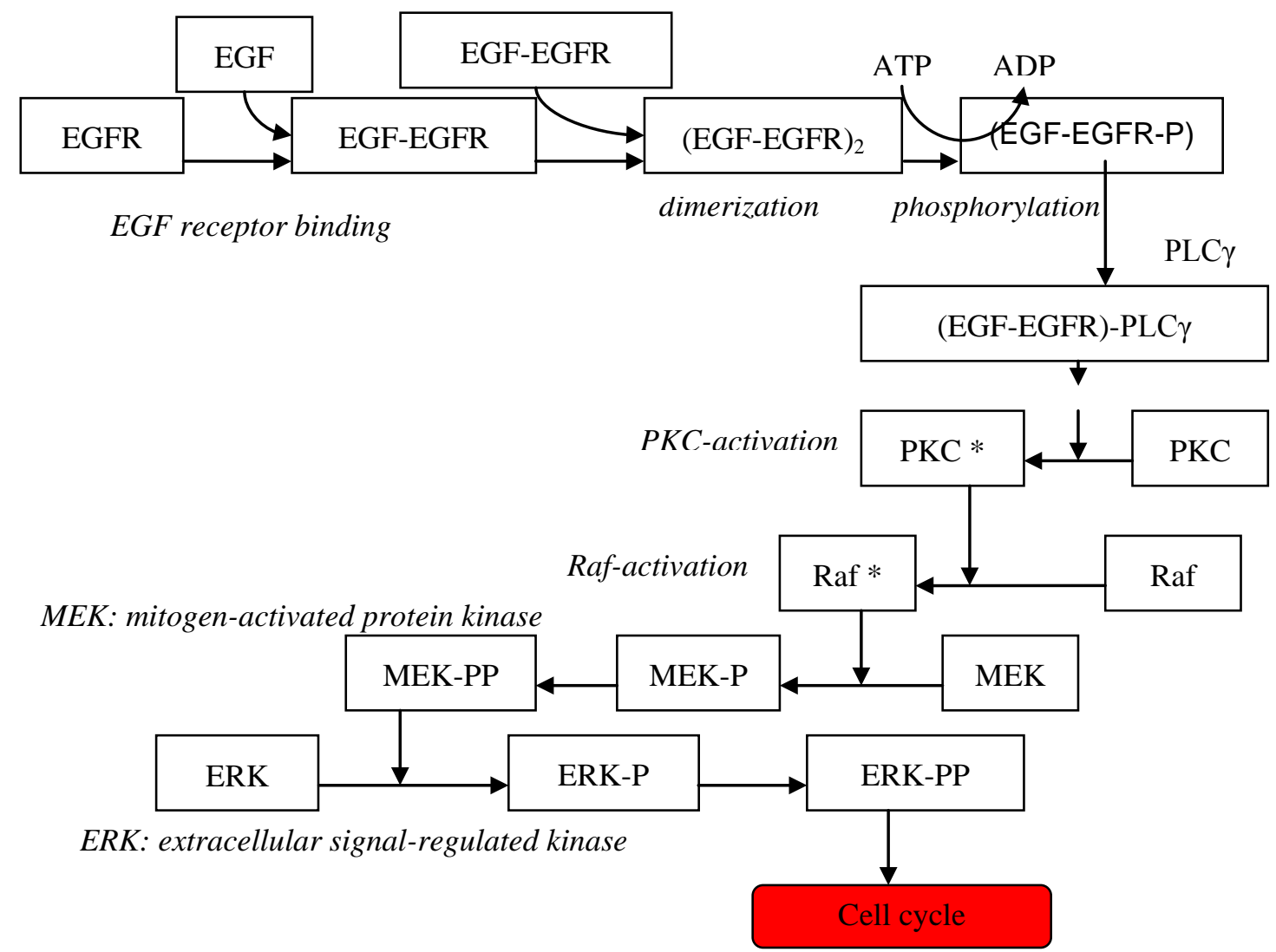

Fig. 2 Downstream signaling pathway triggered by epidermal growth factor receptor (EGFR)(modified after Wang et al. [35]). EGFR is an intrinsic mitogen, that in the presence of an extrinsic chemotactic stimulus (and depending on the cells location) may yield a migration-dominant cell phenotype. The cascade of phosphorylation leads to activation of the extracellular signal-regulated kinase (ERK-PP), resulting in a more effective signal processing in the migration-dominant phenotype (Wang et al. [35]).

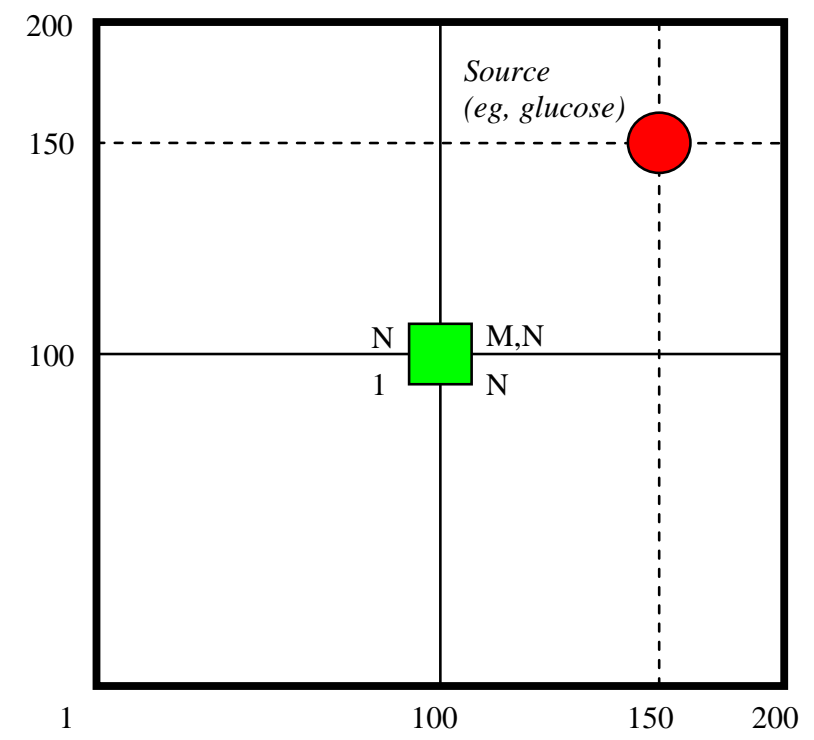

Fig. 3 Modeling of cell migration following a 2-D random walk lattice according to Wang et al. [35]. The migration decision of a cell in a $M \times N$ lattice depends on an attractiveness formula: $T_{i j}=\psi \cdot L_{i j}+(1-\psi) \cdot \varepsilon_{i j}$, with $\psi$ the so-called 'search precision parameter' (for $\psi=0$, a random walk situation is found; for $\psi=1$, the cell selects the location with the highest glucose concentration; $\varepsilon$ is an error term following the normal distribution). 
Whether the opportunistic behavior of disseminating tumor cells is the underlying cause for a lack of scientific interest or not, in multiscale stochastic modeling it plays an indisputable role [4] (see also section below).

\section{The Cell (Survival) Perspective}

Neither cell-free stochastic modeling, nor the theory of so-called cellular automata, so far have satisfactorily resolved the problem of multiscale cancer modeling, although in the case of NSCLC important progress has been made [35]. From the perspective of the abnormal cell, however, a number of pathways are identifiable, each of which influence and in combination they determine the cell survival rate. The cell survival perspective may therefore become a cornerstone for the next generation of multiscale cancer modeling.
From the modeling viewpoint, difficulties arise from the versatility of cell groups during the process of malignant transformation. Practically, annotation systems may benefit from their correspondence with clinically obtained evidence. In this respect, pathological relevance is valued more importantly than mathematical elegance.

Without going into detail too much, the probability for an abnormal cell to develop into a life-threatening cluster of metastases, may result from a number of cell events (cell division, tumor expansion, cell migration, etc.), each of which depending on the occurrence of a set of factors (genetic mismatch, DNA-methylation status, oxygen tension, glucose level, regulators of apoptosis, etc.). Formally, this $P_{\text {survival }}$ is a product of amplitudes $P_{1}{ }^{i}, P_{2}^{j}, . . P_{m}{ }^{n}$ of $m$ successive events at $n$ locations, each of them depending on a different set of occurrencies $\left(O_{m x}, O_{m y}, O_{m z} \ldots\right)$ :

$$
P_{\text {survival }}=P_{1}^{i}\left(O_{\text {gen }}^{i}, O_{\text {meth }}^{i}, O_{\text {oxy }}^{i}, O_{g l u}^{i}\right) \cdot P_{2}^{j}\left(O_{2 x}^{j}, O_{2 y}^{j}, O_{2 z}^{j}, \ldots\right) \ldots P_{m}^{n}=\prod_{m, n} P_{m}^{n}
$$

The stochastic element of the model results from the vaste number of available cells and the capability of these cells to disseminate through the system via different (vascular) routes (see section 2.4). Another major observation from clinical practice is that the prevalence of cancer in the population increases with age. Therefore, in order to integrate the cell survival probabilities for an individual patient, the overall landscape of possible cell states and dissemination routes should reflect an age-dependent element.
In this respect, the notion of DIP (density of information processing) allows for introducing a parameter for the interconnectedness of successive steps in a (neuronal or cellular) signaling network [39]. From this DIP, the notion of "trajectory density" was introduced [6]. (In section 4, we will comment on the notions of "trajectory density" and cell-state landscape for tumor development).

For the overall survival prognosis, the rather unmanageable formula is found:

$$
P_{\text {overall }}\left(x_{\text {age }}, t\right)=1-\sum_{i, j}^{N} \int_{t=0}^{\infty}\left(\prod_{m, n} P_{m}^{n}\right) D I P_{(x, i, t)}\left(1-e^{-\varphi(x, i) . t}\right) d t
$$

In this formula, the integration of the DIP parameter (according to age $x$ of an individual and position $i$ of the $j^{\text {th }}$ route in a cellular network with $N$ nodes) causes the main challenge for making relevant predictions about an individuals prognosis. Also, an age- and network-dependent "fatality" ratio $\varphi(x, i)$, reflecting the impact of (со)morbidity and life-threatening conditions on survival, does not result from modeling but is read from the experienced fact. As a result, $P_{\text {overall }}$ tends to zero for $\lim \mathrm{t} \rightarrow \infty$. Again, evidence from clinical practice will substantiate the (statistically relevant) developmental or pathological transformations in a living cell population. 

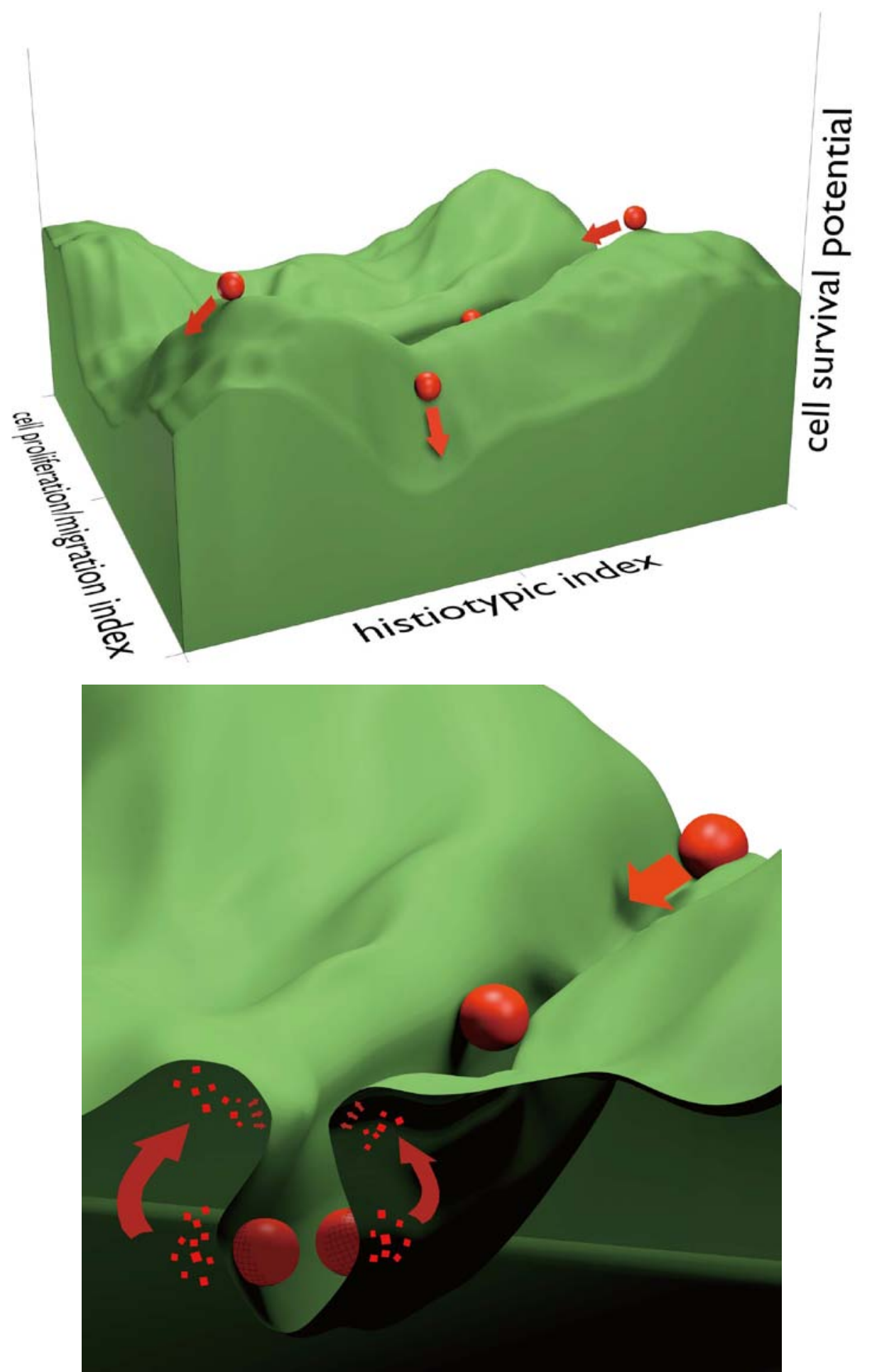

Fig. 4 Theoretical cell-state modeling in a 3-D hyperbody, depending on the cell proliferation/migration potential, a positional and age-dependent histiotypic index and local nematic-isotopic order parameters. Left (overall): Artist impression of a cell-state landscape. The histiotypic index represents an abstract age-dependence of the hyperbody on environmental (effects of high energy radiation on skin cells) or gross anatomical determinants (epi-, [neuro]endo- or mesodermal cues). The proliferation/migration potential is represented according to recent insights in cell-state modeling (reviewed in [7]). Right (detail): Interactions between migrating and resident cells may alter the nematic-isotopic order or cause transient local order parameters, influencing stochastic processes at a local scale. 


\section{Reprogramming and Cell-State Landscape}

The finding that mature cells can be reprogrammed to become pluripotent stem cells [1] echoed into new approaches for studying transformations of mature cell populations. In a recent paper [7], we discussed the abstract notions of defining roadmaps and/or roadblocks in reprogramming [5] and cell-state landscapes obtained from the analysis of density of trajectories [6]. Using a chosen 2D-projection of a hyperbody representing all possible cell-states, on a plane defined by two arbitrary axes and according to a third axis (e.g., pluripotency potential or cell survival potential), one may visualize the possible trajectories of a population of cells. Since cellular development in general does not follow a "linear pattern", as pointed out by the "cancer stem-cell hypothesis" (see section 2.2), more complex patterns may be discerned. For instance, visualization of "ridges", "valleys" and "saddle-points" may indicate a barrier, a furrow or bifurcation point, according to which certain developmental pathways are blocked, guided, split or deviated. However, little is known about the fine-tuning of these processes, caused by local ordering parameters (that may be of nematic, isotopic or transient nature) (Fig. 4).

Of course, the main challenge will be to integrate the experimental evidence obtained from developing immune cell networks or metastasing cell populations into similar cell-state landscapes. The conundrum of these hyperbody visualizations therefore relies on a reproducible, topographically relevant (histiotypically and with respect to local oderering) representation of these biochemical/molecular interaction data. The interaction of some known biochemical pathways and tumor development is well documented (e.g. Wnt-signaling in colon carcinoma [40]). To describe the proliferation-enhancing effect of R-spondin signalling on Lgr5+ stem cells and their association with Wnt-receptor activation, Clevers et al. used the metaphor of am amplifying relay system [41]. So the cancer stem-cell hypothesis may facilitate the introduction of new metaphors, and these metaphors are helpful in visualizing the cellular and molecular processes in an overall non-linear developmental pattern. Another interesting model system is found in the role of the BMPR1B gene (bone morphogenetic protein $[B M P]$ receptor $1 B)$ and epigenetical silencing due to an EZH2 (enhancer of Zeste homolog-2)-dependent mechanism. In this model, EZH2 seems to be involved in methylation of $\mathrm{CpG}$ islands in the BMPR1B promotor, causing initial glioblastoma development and brain tumorigenesis [42, 43].

In the case of NSCLC, studies on the prognostic and predictive uses of KRAS mutations and modification of EGFR signaling are promising [44]. The signaling network downstream of EGFR however is still elusive, containing several possible feedback loops that make the signaling network redundant and, therefore, difficult to read and tackle. Also the role of TTF1 gene products (see section 2.1) in differentiation of lung alveolar Type II cells remains unresolved. Moreover, so far no comprehensive map of all pathways involved in NSCLC has been drawn, let alone sufficient and effective therapies have been developed to cure or contain the disease.

\section{Conclusions}

Multiscale modeling in principle consists of combining biological data that are explained at two or more levels of compartmentalization. For instance, at the level of transcription and transcription regulation, at the level of cellular behavior (proliferation, migration...) or cellular phenotype, the pattern or route followed during metastasis, the development of secondary tumors and co-morbidity mechanisms. Some of these aspects of developmental or pathological transformation have been clarified according to deterministic studies (classically defined according to cause-and-effect relationships), others may be explained following probabilistic (or stochastic) 
modeling. The latter aspects are locally recognized by random effects or by dynamical non-linear interactions at a larger distance.

Although the group of NSCLC (non-small cell lung cancer) has a fragmentated appearance, due to genetic background and divergent pathological processes taking place, the disease became a target for multiscale modeling studies. In this paper, it is suggested that some visualization techniques used in cell-state modeling through reprogramming $[1,2,5,6]$ may become useful too in modeling tumor expansion and metastasis. However, more experimental studies and access to clinical data will be needed to integrate the different aspects of the disease into a comprehensive multiscale model. For instance, whether and how the over-expression (and/or amplification) of the TTF1 gene in different adenocarcinomas is linked to cell cycle propagation. Another example is the well known relation between EGFR over-expression and increased metastasis; the interaction with other pathways in this multifaceted dragon, however, remains a challenge to multiscale modeling.

\section{References}

[1] Takahashi, K., and Yamanaka, S. 2006. "Induction of Pluripotent Stem Cells from Mouse Embryonic and Adult Fibroblast Cultures by Defined Factors.” Cell 126 (4): 663-76.

[2] Takahashi, K., Tanabe, K., Ohnuki, M., Narita, M., Ichisaka, T., Tomoda, K., and Yamanaka, S. 2007. "Induction of Pluripotent Stem Cells from Adult Human Fibroblasts by Defined Factors.” Cell 131 (5): 861-72.

[3] Driessens, G., Beck, B., Caauwe, A., Simons, B. D., and Blanpain, C. 2012. "Defining the Mode of Tumour Growth by Clonal Analysis.” Nature 448 (7412): 527-31.

[4] Nguyen, D. X. 2011. "Tracing the Origins of Metastasis." Journal of Pathology 223 (2): 195-204.

[5] Polo, J. M., Anderssen, E., Walsh R. M., Schwarz, B. A., Nefzger, C. M., Lim, S. M., Borkent, M., Apostolou, E., Alaei, S., Cloutier, J., Bar-Nur, O., Cheloufi, S., Stadtfeld, M., Figueroa, M. E., Robinton, D., Natesan, S., Melnick, A., Zhu, J., Ramaswamy, S., and Hochedlinger, K. 2012. “A Molecular Roadmap of Reprogramming Somatic Cells into iPS Cells.” Cell 151 (7): 1617-32.

[6] Shu, J., Wu, C., Wu, Y., Li, Z., Shao, S., Zhao, W., Tang, X., Yang, H., Shen, L., Zuo, X., Yang, W., Shi, Y., Chi, X.,
Zhang, H., Gao, G., Shu, Y., Yuan, K., He, W., Tang, C., Zhao, Y., and Deng, H. 2013. "Induction of Pluripotency in Mouse Somatic Cells with Lineage Specifiers.” Cell 153 (5): 963-75.

[7] Allaerts, W. 2014. “Annotation Grids for Cell Lineage and Tumor Metastasis: II. Defining the Architecture for Multiscale Probabilistic and Deterministic Models.” Medical Hypotheses and Research 9 (1): 1-15.

[8] Edelman, G. M. 1988. Topobiology. An Introduction to Molecular Embryology. New York: Basic Books Inc.

[9] Abbas, A. K., and Lichtman, A. H. ed. 2005. Cellular and Molecular Immunology. Philadelphia: Elsevier Saunders.

[10] van Dongen, J. J. M., Macintyre, E. A., Gabert, E. A., Delabesse, E., Rossi, V., Saglio, G., Gottardi, E., Rambaldi, A., Dotti, G., Griesinger, F., Parreira, A., Gemeiro, P., González Diáz, M., Malec, M., Langerak, A. W., San Miguel, J. F., and Biondi, A. 1999. "Standardized RT-PCR Analysis of Fusion Gene Transcripts from Chromosome Aberrations in Acute Leukemia for Detection of Minimal Residual Disease. (Report of Biomed-1 Concerted Action).” Leukemia 13 (12): 1901-28.

[11] Berghmans, T., Paesmans, M., Mascaux, C., Martin, B., Meert, A. P., Haller, A., Lafitte, J. J., and Sculier, J. P. 2006. "Thyroid Transcription Factor 1 -a New Prognostic Factor in Lung Cancer: A Meta-analysis.” Annals of Oncology 17 (11): 1673-6.

[12] Perner, S., Wagner, P. L., Soltermann, A., LaFargue, C., Tischler. V., Weir. B. A., Weder. W., Meyerson. M., Giordano. T. J., Moch. H., and Rubin, M. A. 2009. “TTF1 Expression in Non-Small Cell Lung Carcinoma: Association with TTF1 Gene Amplification and Improved Survival.” Journal of Pathology 217 (1): 65-72.

[13] Boggaram, V. 2009. "Thyroid Transcription Factor-1 (TTF-1/Nkx2.1/TITF1) Gene Regulation in the Lung.” Clinical Science 116 (1): 27-35.

[14] Civitareale, D., Lonigro, R., Sinclair, A. J., and Di Lauro, R. 1989. "A Thyroid-Specific Nuclear Protein Essential for Tissue-Specific Expression of the Thyroglobulin Promoter.” EMBO Journal 8 (9): 2537-42.

[15] Civitareale, D., Castelli, M. P., Falasca, P., and Saiardi, A. 1993. "Thyroid Transcription Factor 1 Activates the Promoter of the Thyrotropin Receptor Gene.” Molecular Endocrinology 7 (12): 1589-95.

[16] Abramowicz, M. J., Vassart, G., and Christophe, D. 1992. "Functional Study of the Human Thyroid Peroxidase Gene Promoter.” European Journal of Biochemistry 203 (3): 467-73.

[17] Ramirez, M. I., Rishi, A. K., Cao, Y. X., and Williams, M. C. 1997. "TGT3, Thyroid Transcription Factor I, and Sp1 Elements Regulate Transcriptional Activity of the 1.3-Kilobase Pair Promoter of T1 $\alpha$, a Lung Alveolar Type 
I Cell Gene.” Journal of Biological Chemistry 272 (42): 26285-94.

[18] Bruno, M. D., Bohinski, R. J., Huelsman, K. M., Whitsett, J. A., and Korfhagen, T. R. 1995. "Lung Cell-Specific Expression of the Murine Surfactant Protein A (SP-A) Gene Is Mediated by Interactions between the SP-A Promoter and Thyroid Transcription Factor-1.” Journal of Biological Chemistry 270 (12): 6531-6.

[19] Bohinski, R. J., Di Lauro, R., and Whitsett, J. A. 1994. “The Lung-Specific Surfactant Protein B Gene Promoter Is a Target for Thyroid Transcription Factor 1 and Hepatocyte Nuclear Factor 3, Indicating Common Factors for Organ-Specific Gene Expression along the Foregut Axis.” Molecular and Cellular Biology 14 (9): 5671-81.

[20] Kelly, S. E., Bachurski, C. J., Burhans, M. S., and Glasser, S. W. 1996. "Transcription of the Lung-Specific Surfactant Protein C Gene Is Mediated by Thyroid Transcription Factor 1.” Journal of Biological Chemistry 271 (12): 6881-8.

[21] Toonen, R. F., Gowan, S., and Bingle, C. D. 1996. "The Lung Enriched Transcription Factor TTF-1 and the Ubiquitously Expressed Proteins Sp1 and Sp3 Interact with Elements Located in the Minimal Promoter of the Rat Clara Cell Secretory Protein Gene.” Biochemical Journal 316 (Pt 2): 467-73.

[22] Besnard, V., Xu, Y., and Whitsett, J. A. 2007. "Sterol Response Element Binding Protein and Thyroid Transcription Factor-1 (Nkx2.1) Regulate Abca 3 Gene Expression.” American Journal of Physiology Lung Cellular and Molecular Physiology 293 (6): L1395-405.

[23] Zhou, L., Lim, L., Costa, R. H., and Whitsett, J. A. 1996. “Thyroid Transcription Factor-1, Hepatocyte Nuclear Factor-3 $\beta$, Surfactant Protein B, C, and Clara Cell Secretory Protein in Developing Mouse Lung." Journal of Histochemistry and Cytochemistry 44 (10): 1183-93.

[24] Sparkman, L., Chandru, H., and Boggaram, V. 2006. "Ceramide Decreases Surfactant Protein B Gene Expression via Downregulation of TTF-1 DNA Binding Activity.” American Journal of Physiology Lung Cellular and Molecular Physiology 290 (2): L351-8.

[25] Tanaka, H., Yanagisawa, K., Shinjo, K., Taguchi, A., Maeno, K., Tomida, S., Shimada, Y., Osada, H., Kosaka, T., Matsubara, H., Mitsudomi, T., Sekido, Y., Tanimoto, M., Yatabe, Y., and Takahashi, T. 2007. "Lineage-Specific Dependency of Lung Adenocarcinomas on the Lung Development Regulator TTF-1.” Cancer Research 67 (13): 6007-11.

[26] Lin, C., Song, H., Huang, C., Yao, E., Gacayan, R., Xu, S. M., and Chuang, P. T. 2012. “Alveolar Type II Cells Possess the Capability of Initiating Lung Tumor Development.” PloS ONE 7 (12): e53817.

[27] Barkauskas, C. E., Cronce, M. J, Rackley, C. R., Bowie, E.
J., Keene, D. R., Stripp, B. R., Randell, S. H., Noble, P. W., and Hogan, B. L. M. 2013. "Type 2 Alveolar Cells Are Stem Cells in Adult Lung." Journal of Clinical Investigation 123 (7): 3025-36.

[28] Lapidot, T., Sirard, C., Vormoor, J., Murdoch, B., Hoang, T., Caceres-Cortes, J., Minden, M., Paterson, B., Caligiuri, M.A., and Dick, J. E. 1994. “A Cell Initiating Human Acute Myeloid Leukaemia after Transplantation into SCID Mice.” Nature 367 (6464): 645-648.

[29] Hackel, P. O., Zwick, E., Prenzel, N., and Ullrich, A. 1999. "Epidermal Growth Factor Receptors: Critical Mediators of Multiple Receptor Pathways.” Current Opinion in Cell Biology 11 (2): 184-9.

[30] Hirsch, F. R., Varella-Garcia, M., Bunn, P. A. Jr., Di Maria, M. V., Veve, R., Bremmes, R. M., Baron, A. E., Zeng, C., and Franklin, W. A. 2003. "Epidermal Growth Factor Receptor in Non-Small-Cell Lung Carcinomas: Correlation between Gene Copy Number and Protein Expression and Impact on Prognosis.” Journal of Clinical Oncology 21 (20): 3798-807.

[31] Ciardiello, F., De Vita, F., Orditura, M., and Tortora, G. 2004. "The Role of EGFR Inhibitors in Nonsmall Lung Cancer.” Current Opinion in Oncology 16 (2): 130-5.

[32] Putnam, E. A., Yen, N., Gallick, G. E., Steck, P. A., Fang, K., Akpakip, B., Gazdar, A. F., and Roth, J. A. 1992. "Autocrine Growth Stimulation by Transforming Growth Factor-alpha in Human Non-Small Cell Lung Cancer." Surgical Oncology 1 (1): 49-60.

[33] Rusch, V., Baselga, J., Cordon-Cardo, C., Orazem, J., Zaman, M., Hoda, S., McIntosh, J., Kurie, J., and Dmitrovsky, E. 1993. "Differential Expression of the Epidermal Growth Factor Receptor and its Ligands in Primary Non-Small Cell Lung Cancers and Adjacent Benign Lung.” Cancer Research 53 (10 Suppl): 2379-85.

[34] Price, J. T., Wilson, H. M., and Haites, N. E. 1996. "Epidermal Growth Factor (EGF) Increases the in vitro Invasion, Motility and Adhesion Interactions of the Primary Renal Carcinoma Cell Line, A704.” European Journal of Cancer 32 (11): 1977-82.

[35] Wang, Z., Zhang, L., Sagotsky, J., and Deisboeck, T. S. 2007. "Simulating Non-Small Cell Lung Cancer with a Multiscale Agent-Based Model.” Theoretical Biology and Medical Modelling 4 (1): 50-63.

[36] Onuigbo, W. I. B. 1963. "A Modified Theory of Retrograde Lymphatic Metastasis in Lung Cancer." British Journal of Diseases of the Chest 57 (3): 120-5.

[37] Batson, O. V. 1957. "The Vertebral Vein System.” American Journal of Roentgenology 78 (2): 195-212.

[38] Williams, A., Newell, R. L. M., and Collins, P., ed. 2005. "Back and Macroscopic Anatomy of Spinal Cord.” In Gray's Anatomy, edited by Standring, S. Edinburgh, London, New York, Sydney, Toronto: Churchill 
Livingstone, Elsevier Ltd, 725-98.

[39] Ma’ayan, A., Jenkins, S. L., Neves, S., Hasseldine, A., Grace, E., Dubin-Thaler, B., Eungdamrong, N. J., Weng, G., Ram, P. T., Rice, J. J., Kershenbaum, A., Stolovitzky, G. A., Blitzer, R. D., and Iyengar, R. 2005. "Formation of Regulatory Patterns during Signal Propagation in a Mammalian Cellular Network.” Science 309 (5737): 1078-83.

[40] Korinek, V., Barker, N., Morin, P. J., van Wichen, D., de Weger, R., Kinzler, K. W., Vogelstein, B., and Clevers, H. 1997. "Constitutive Transcriptional Activation by a Beta-Catenin-Tcf Complex in APC -/- Colon Carcinoma.” Science 275 (5307): 1784-7.

[41] De Lau, W., Barker, N., Low, T. Y., Koo, B. K., Li, V. S., Teunissen, H., Kujala, P., Haegebarth, A., Peters, P. J., van de Wetering, M., Stange, D. E., van Es, J. E., Guardavaccaro, D., Schasfoort, R. B., Mohri, Y., Nishimori, K., Mohammed Sheck, A. J., and Clevers, H.
2011. "Lgr5 Homologues Associate with Wnt Receptors and Mediate R-Spondin Signalling.” Nature 476 (7360): 293-7.

[42] Lee, J., Son, M. J., Woolard, K., Donin, N. M., Li, A., Cheng, C. H., Kotliarova, S., Kotliarov, Y., Walling, J., Ahn, S., Kim, M., Totonchy, M., Cusack, T., Ene, C., Ma, H., Su, Q., Zenklusen, J. C., Zhang, W., Maric, D., and Fine, H. A. 2008. "Epigenetic-Mediated Dysfunction of the Bone Morphogenetic Protein Pathway Inhibits Differentiation of Glioblastoma-Initiating Cells.” Cancer Cell 13 (1): 69-80.

[43] Allaerts, W. 2011. "New Cellular Concepts in Neuroendocrinology: the Anterior Pituitary Case." Current Trends in Endocrinolog, 5: 19-28.

[44] Martin, P., Leighl, N. B., Tsao, M. S., and Shepherd, F. A. 2013. "KRAS Mutations as Prognostic and Predictive Markers in Non-Small Cell Lung Cancer.” Journal of Thoracic Oncology 8 (5): 530-42. 